\title{
ON THE LOCUS OF THE FOCI OF CONICS HAVING DOUBLE CONTACT WITH TWO FIXED CONICS.
}

\author{
BY R. A. ROBERTS.
}

THE system of conics having double contact with two conics includes as special cases the two systems of conics passing through four fixed points and touching four fixed lines, respectively. These latter result from making the fixed conics break up into factors, in the first case, in the tangential sense, and in the second case directly. Thus the general case includes and combines together into one whole the properties of the two special cases. Now I propose to consider the locus of the foci of the general system. This must include as special cases the known loci in the case of conics through four fixed points and touching four fixed lines. The first is a curve of the sixth order, which, when the points are concyclic, as has been shown by Professor Sylvester, breaks up into the two circular cubics having the points for foci, and the second is a circular cubic having its double focus on itself and passing through all the intersections of the four lines.

Now foci, being the intersections of tangents drawn to the curve from the imaginary points at infinity, are most easily treated by tangential coördinates, which I consequently use, the equations being precisely the same as the direct ones; viz. as given by Salmon, a conic having double contact with $S, S^{\prime}$ is, if $S-k S^{\prime}=E F$;

$$
\theta^{2} E^{2}-2 \theta\left(S+k S^{\prime}\right)+F^{2}=0 .
$$

But this being now a tangential equation, we may put

$$
\begin{gathered}
E=\lambda x_{1}+\mu y_{1}+v, \\
F=\lambda x_{2}+\mu y_{2}+v, \\
S+k S^{\prime}=A \lambda^{2}+B \mu^{2}+C \nu^{2}+2 F \mu^{2}+2 G \nu \lambda+2 H \lambda \mu,
\end{gathered}
$$

where $x, y$ are rectangular Cartesian coördinates. Now the foci are the points of intersection of the tangents drawn parallel to $x \pm i y$. Hence for the foci we may put $v=x \pm i y, \lambda=-1$, $\mu=\mp i$; we thus get

$$
\begin{aligned}
& \theta^{2}(u-a)^{2}-2 C \theta(u-c)(u-d)+(u-b)^{2}=0, \\
& \theta^{2}\left(v-a^{\prime}\right)^{2}-2 C \theta\left(v-c^{\prime}\right)\left(v-d^{\prime}\right)+\left(v-b^{\prime}\right)^{2}=0,
\end{aligned}
$$

where $u=x+i y, v=x-i y$, and $a, b, c, d$ are values of $u$ for certain points, $E, F$, and two others, the corresponding values 
of $v$ being $a^{\prime}, b^{\prime}, c^{\prime}, d^{\prime}$. Hence, by elimination of $\theta$, the locus is found to be, in general, a curve of the sixth degree, which may be written

$$
\begin{aligned}
4\left\{(u-a)^{2}(u-b)^{2}-C^{2}(u-c)^{2}(u-d)^{2}\right\} & \\
& \times\left\{\left(v-a^{\prime}\right)^{2}\left(v-b^{\prime}\right)^{2}-C^{2}\left(v-c^{\prime}\right)^{2}\left(v-d^{\prime}\right)^{2}\right\} \\
= & \left\{(u-a)^{2}\left(v-b^{\prime}\right)^{2}+(u-b)^{2}\left(v-a^{\prime}\right)^{2}\right. \\
& \left.-2 C^{2}(u-c)(u-d)\left(v-c^{\prime}\right)\left(v-d^{\prime}\right)\right\}^{2}, \\
\text { or } \quad 4 C^{2} & \left\{(u-c)(u-d)\left(v-b^{\prime}\right)^{2}-\left(v-c^{\prime}\right)\left(v-d^{\prime}\right)(u-b)^{2}\right\} \\
& \times\left\{(u-c)(u-d)\left(v-a^{\prime}\right)^{2}-\left(v-c^{\prime}\right)\left(v-d^{\prime}\right)(u-a)^{2}\right\} \\
= & \left\{(u-a)^{2}\left(v-b^{\prime}\right)^{2}-(u-b)^{2}\left(v-a^{\prime}\right)^{2}\right\}^{2} .
\end{aligned}
$$

From the latter equation it is manifest that the locus is of the sixth degree, as in each of the factors the coefficient of $u^{2} v^{2}$ is seen to vanish; and, moreover, that the circular points at infinity, as well as the points $E, F$, are double points on the curve. From the same equation it is easy to see that

$$
\frac{(u-a)^{2}}{\left(v-a^{\prime}\right)^{2}}=\frac{(u-b)^{2}}{\left(v-b^{\prime}\right)^{2}}=\frac{(u-c)(u-d)}{\left(v-c^{\prime}\right)\left(v-d^{\prime}\right)}
$$

must also give double points, and indeed it is evident that the values of $u, v$ which make the quadratics in $\theta$ identical must lead to such points.

Hence to find these nodes, we may write

$$
\begin{gathered}
(u-a)^{2}=k\left(v-a^{\prime}\right)^{2},(u-b)^{2}=k\left(v-b^{\prime}\right)^{2}, \\
(u-c)(u-d)=k\left(v-c^{\prime}\right)\left(v-d^{\prime}\right) .
\end{gathered}
$$

Thus if $l, m, n$ are any numbers,

$$
\begin{aligned}
l(u-a)^{2}+m & (u-b)^{2}+n(u-c)(u-d) \\
& =k\left\{l\left(v-a^{\prime}\right)^{2}+m\left(v-b^{\prime}\right)^{2}+n\left(v-c^{\prime}\right)\left(v-d^{\prime}\right)\right\},
\end{aligned}
$$

so that by properly selecting $l, m, n$, we obtain

$$
\begin{gathered}
P u+Q=k C_{1}, \\
C_{2}=k\left(P^{\prime} v+Q^{\prime}\right),
\end{gathered}
$$

whence

$$
(P u+Q)\left(P^{\prime} v+Q^{\prime}\right)=C_{1} C_{2},
$$

which represents a circle. But we also have

$$
(u-a)^{2}\left(v-b^{\prime}\right)^{2}-(u-b)^{2}\left(v-a^{\prime}\right)^{2}=0,
$$


which breaks up into the line joining $E, F$, and the circle described on $E F$ as diameter. One point on $E F$ is irrelevant, so that three nodes are obtained in this way.

Now proceeding to the first form of the equation of the locus, we see that the lines

$$
\begin{aligned}
& (u-a)^{2}(u-b)^{2}-C^{2}(u-c)^{2}(u-d)^{2}=0, \\
& \left(v-a^{\prime}\right)^{2}\left(v-b^{\prime}\right)^{2}-C^{2}\left(v-c^{\prime}\right)^{2}\left(v-d^{\prime}\right)^{2}=0
\end{aligned}
$$

are bitangents of the locus ; that is, according to the definition of a focus, the intersections of the first four lines with the second four determine the foci of the curve, and, further, these points are not merely ordinary foci, but double foci of a certain kind. Now it is easily seen that

$$
\begin{aligned}
& (u-a)(u-b) \pm C(u-c)(u-d)=0, \\
& \left(v-a^{\prime}\right)\left(v-b^{\prime}\right) \pm C\left(v-c^{\prime}\right)\left(v-d^{\prime}\right)=0,
\end{aligned}
$$

are precisely the equations which determine the foci of the given conics $S, S^{\prime}$.

Hence the foci of the two fixed conics are double foci of the locus. But there are also other foci, which are of the ordinary kind, as we can see by the following considerations: the locus has seven double points ; therefore by the formula

$$
m=n(n-1)-2 \delta ;
$$

taking $n=6, \delta=7$, we see that the class of the locus is 16 , so that 12 tangents can be drawn to the curve from the double point $I$. But the four lines to the double foci are bitangents, and count as eight ordinary tangents. Thus, four ordinary tangents can be drawn to the curve from $I$, and these give rise to four ordinary foci.

To find the points at infinity on the curve, we make $u$ or $v$ infinite in (1), and we thus get

$$
\theta^{2}-2 C \theta+1=0 .
$$

This determines the two curves of the system which touch the line at infinity, or, in other words, are parabolas, as ought evidently to be the case from geometrical considerations. Hence if these two parabolas are real, the locus has two real asymptotes.

Since there are three values of $k$ for which $S-k S^{\prime}$ breaks up into factors, there are, as shown in Salmon's Conics, three systems of conics having double contact with $S$, and the determination of the locus of the foci is precisely the same in each case; that is, the entire locus of the foci consists, in general, of three sextic curves. 
We now consider some cases in which the locus reduces to one of lower degree.

Solving the equations (1), we get

$$
\begin{aligned}
& u=\frac{a \theta^{2}+C(c+d) \theta+b \pm \sqrt{\Theta_{1}}}{\theta^{2}-2 C \theta+1}, \\
& v=\frac{a^{\prime} \theta^{2}+C\left(c^{\prime}+d^{\prime}\right) \theta+b^{\prime} \pm \sqrt{\Theta_{2}}}{\theta^{2}-2 C \theta+1},
\end{aligned}
$$

where $\Theta_{1}=\theta\left\{2 C(a-c)(a-d) \theta^{2}+2 C(b-c)(b-d)\right.$

$$
\begin{aligned}
\Theta_{2}=\theta\left\{2 C\left(a^{\prime}-c^{\prime}\right)\left(a^{\prime}-d^{\prime}\right) \theta^{2}\right. & {\left.\left.\left[(a-b)^{2}-C^{2}(c-d)^{2}\right] \theta\right\}, c^{\prime}\right)\left(b^{\prime}-d^{\prime}\right) } \\
+ & {\left.\left[\left(a^{\prime}-b^{\prime}\right)^{2}-C^{2}\left(c^{\prime}-d^{\prime}\right)^{2}\right] \theta\right\} . }
\end{aligned}
$$

From these expressions it is evident that the locus will simplify if $\Theta_{1}$ and $\Theta_{2}$ are proportional to each other. Now if we consider the geometrical meaning of $\Theta_{1}=0$, it is easy to see that the quadratic factor gives the two conics of the system which may be described to pass through the point $I$. Similarly, $\Theta_{2}=0$ gives the two conics through $J$. Hence if the two quadratic factors are proportional, two conics of the system pass through $I, J$, or, in other words, are circles. Now this condition will evidently be satisfied if the two fixed conics have a principal axis in common. Thus, there are two circles belonging to the system

$$
\theta^{2}(x+h)^{2}+(x+k)^{2}-2 \theta\left(a x^{2}+b y^{2}+2 g x+c\right)=0,
$$

which are evidently determined by

$$
\theta^{2}+1-2 \theta(a-b)=0 \text {. }
$$

It is evident that the locus must be divisible by the equation of the principal axis, or rather its square, the remaining factor giving the locus of the foci lying on the other principal axis; namely, the axis major for real foci.

Taking, then, the fixed principal axis as the axis of $y$ in the equations (2), we have $a^{\prime}=a, b^{\prime}=b, c^{\prime}=c, d^{\prime}=d$, and since

$$
\begin{aligned}
u+v & =2 x, u-v=2 i y, \\
x & =\frac{a \theta^{2}+C(c+d) \theta+b}{\theta^{2}-2 C \theta+1}, \\
y & =\frac{\sqrt{-\Theta}}{\theta^{2}-2 C^{2} \theta+1}
\end{aligned}
$$

whence, also, $\quad x^{2}+y^{2}=\frac{a^{2} \theta^{2}-2 C c d \theta+b^{2}}{\theta^{2}-2 C \theta+1}$. 
Thus eliminating $\theta$, we get a result of the form

$$
\left(x^{2}+y^{2}\right)^{2}+(p x+q)\left(x^{2}+y^{2}\right)+r x^{2}+2 s x+t=0,
$$

which represents a bicircular quartic of which $y=0$ is an axis of symmetry. A particular case that may be considered as meriting special consideration is when the two fixed conics are confocal, and the enveloping conics are concentric with them. If a line $\lambda x+\mu y+\nu=0$ touches

$$
\frac{x^{2}}{a^{2}}+\frac{y^{2}}{b^{2}}-1=0
$$

we have

$$
a^{2} \lambda^{2}+b^{2} \mu^{2}-v^{2}=0,
$$

and for the second conic, say, $a^{12} \lambda^{2}+b^{12} \mu^{2}=\nu^{2}$, where

$$
a^{2}-a^{\prime 2}=b^{2}-b^{2}=h^{2} .
$$

Then the concentric system of enveloping conics may be written $h^{2} \cos \theta\left(\lambda^{2}-\mu^{2}\right)+2 h^{2} \sin \theta \lambda \mu=\left(\alpha^{2}+\alpha^{\prime 2}\right) \lambda^{2}+\left(b^{2}+b^{\prime 2}\right) \mu^{2}-2 \nu^{2}$. Hence for the foci, putting $\lambda=1, \mu=i, \nu=-(x+i y)$, we get

$$
x^{2}-y^{2}-c^{2}+h^{2} \cos \theta=0,2 x y+h^{2} \sin \theta=0 ;
$$

thus the locus is the Cassinian oval

$$
\left(x^{2}+y^{2}\right)^{2}-2 c^{2}\left(x^{2}-y^{2}\right)+c^{4}-h^{4}=0 .
$$

This equation, of course, also denotes that the product of the distances of a focus of the variable conic from the two fixed foci is constant.

There is also another case in which two conics of the system are circles. Consider the system

$$
\theta^{2} P^{2}+Q^{2}-\theta\left(S+k S^{\prime}\right)=0 ;
$$

then if $P, Q$ are right angles to each other, and parallel to the axes of $S+k S^{\prime}$, there are two circles of the system; for, putting $P=x, Q=y$, and

$$
S+k S^{\prime}=a x^{2}+b y^{2}+2 g x+2 f y+c,
$$

two circles are evidently given by the roots of the equation

$$
\theta^{2}-1-\theta(a-b)=0 \text {. }
$$

The two fixed conics being in this case

$$
a x^{2}+b y^{2} \pm 2 x y+2 g x+2 f y+c=0,
$$


two chords of intersection, $x, y$, are rectangular, and, further, the eccentricities of both are equal, as the asymptotes, real or imaginary, contain equal angles.

Now the principal axes of

$$
\theta^{2} x^{2}+y^{2}-\theta\left(a x^{2}+b y^{2}+2 g x+2 f y+c\right)=0
$$

are evidently obtained by differentiating with regard to $x$ and $y$; i.e. are

$$
\begin{aligned}
& (\theta-a) x-\theta g=0, \\
& (1-\theta b) y-f=0,
\end{aligned}
$$

and according as we use one or other of these equations we must obtain different loci. Hence as the complete locus is a circular curve of the sixth degree, the separate loci must be circular cubics, as could also be demonstrated from (2), when $\Theta_{1}, \Theta_{2}$ are supposed to be proportional.

Now let us consider the case in which one of the fixed conics is a circle.

One of the equations (1) which determine the foci of the locus may be written

$$
\{\theta(u-a)+u-b\}^{2}=2 \theta\{(u-a)(u-b)+C(u-c)(u-d)\},
$$

the factors on the right side giving the foci of one of the fixed conics. Hence, if the latter is a circle, the factor must be a perfect square; that is, we may put

$$
(u-a)(u-b)+C(u-c)(u-d)=2 m^{2}(u-e)^{2} .
$$

Thus, if $\theta=t^{2}$, we have

and similarly

$$
t^{2}(u-a)+u-b \pm 2 m t(u-e)=0,
$$

$$
\begin{aligned}
& t^{2}\left(v-a^{\prime}\right)+v-b^{\prime} \pm 2 m t\left(v-e^{\prime}\right)=0, \\
& u=\frac{a t^{2}+b \pm 2 m e t}{t^{2}+1 \pm 2 m t}, \\
& v=\frac{a^{\prime} t^{2}+b^{\prime} \pm 2 m e t}{t^{2}+1 \pm 2 m t} .
\end{aligned}
$$

If we take the same signs of $t$ in $u$ and $v$, we evidently get a conic; and the foci of this conic are easily seen to coincide with the foci of the fixed conic. But if we take different signs of $t$, viz. if we write

$$
\begin{aligned}
& u=\frac{a t^{2}+b+2 m e t}{t^{2}+1+2 m t}, \\
& v=\frac{a^{\prime} t^{2}+b^{\prime}-2 m e t}{t^{2}+1-2 m t}
\end{aligned}
$$


we obtain a unicursal bicircular quartic which has also the foci of the fixed conic as foci.

A direct method of considering the locus in this case may be worth considering. Let the tangential equation of the circle be

$$
(\alpha \lambda+\beta \mu+\gamma \nu)^{2}-r^{2}\left(\lambda^{2}+\mu^{2}\right)=0,
$$

or $G^{2}-r^{2} \Omega=0$, then we may take

$$
(\theta E+F)^{2}-4 \theta\left(G^{2}-r^{2} \Omega\right)=0
$$

as the tangential equation of the system of conics. Now we find the foci of a conic by combining its equation with the equation of the circular points, viz. $\Omega=0$, so as to obtain an equation which breaks up into factors, these factors giving the foci. But putting $\Omega=0$, we get

$$
(\theta E+F)^{2}-4 \theta G^{2}=0,
$$

which breaks up into the factors

$$
\theta E+F \pm 2 G \sqrt{\theta}=0
$$

If these are real, they give the real foci, and the envelope with regard to $\theta$ gives the locus of the foci in tangential coordinates. Thus the locus is $G^{2}=E F$, a conic passing through $E, F, G$ being the intersection of the tangents at these points. Now $G^{2}-r^{2} \Omega=E F$ is the fixed base conic, and $G^{2}-E F=0$ is confocal with this, so that the locus is a conic confocal with the base conic, as we have seen already. But further, this conic is now shown to pass through the points $E, F$. Thus for the three systems of variable conics the locus consists of three conics confocal with the given conic and passing, respectively, through a pair of the intersections of the common tangents of the given conic and circle.

It may be observed that Chasles originally demonstrated the theorem that the intersections of the common tangents of a conic and a circle lie by pairs on conics confocal with the given conic.

This method only gives the locus of the foci when (3) represents real foci. If these factors give imaginary foci, then the locus of the real foci consists of nodal bicircular quartics, as has been shown already.

If the two conics are circles, then by the preceding results we can easily deduce the locus of the foci. We thus find that if a conic has double contact with two fixed circles, $S, S^{\prime}$, the locus of the foci consists of a pair of circles concentric with $S$, another pair concentric with $S^{\prime}$, a circle coaxial with $S, S^{\prime}$, and the line joining the centres of $S, S^{\prime}$. 
These results may be easily proved directly.

Let

$$
\frac{x^{2}}{a^{2}}+\frac{y^{2}}{b^{2}}-1=0
$$

be the conic referred to its axes, and let

$$
\begin{aligned}
& (x-\alpha)^{2}+y^{2}=r^{2}, \\
& x^{2}+(y-\beta)^{2}=\rho^{2},
\end{aligned}
$$

be circles having double contact with the conic. Then it can be easily shown that

$$
\frac{\alpha^{2}}{c^{2}}+\frac{r^{2}}{b^{2}}=1, \quad \frac{\rho^{2}}{a^{2}}-\frac{\beta^{2}}{c^{2}}=1
$$

where $c^{2}=a^{2}-b^{2}$

Now if we take two circles of the first system, the foci simply lie on the line joining their centres; but if the circles are both of the second system, we have $\rho^{2}=\frac{a^{2}}{c^{2}}\left(\beta^{2}+c^{2}\right)=\frac{a^{2}}{c^{2}} R^{2}$, where $R$ is the distance of a focus from the centre of one of the circles; also, $\rho^{\prime 2}=\frac{a^{2}}{c^{2}} R^{\prime 2}$ for another circle. Hence $\frac{R^{2}}{\rho^{2}}=\frac{R^{\prime 2}}{\rho^{\prime 2}}$, which may be written

$$
\frac{R^{2}-\rho^{2}}{\rho^{2}}-\frac{\left(R^{\prime 2}-\rho^{\prime 2}\right)}{\rho^{\prime 2}}=0
$$

showing that the locus is a circle coaxial with the two given circles.

If the two circles are of different systems from

$$
\begin{aligned}
& \frac{\alpha^{2}}{c^{2}}+\frac{r^{2}}{b^{2}}=1, \quad \frac{\rho^{2}}{\alpha^{2}}-\frac{\beta^{2}}{c^{2}}=1, \\
& \frac{\alpha^{2}+\beta^{2}}{c^{2}}=\frac{d^{2}}{c^{2}}=\frac{\rho^{2}}{a^{2}}-\frac{r^{2}}{b^{2}},
\end{aligned}
$$

we get

if $d$ is the distance between the centres of the circles. Hence, $e$ being the eccentricity, we have

$$
d^{2}=e^{2} \rho^{2}-\frac{e^{2}}{1-e^{2}} r^{2},
$$

so that $e^{2}$ is determined by a quadratic equation. Now we have $R^{2}=e^{2} \rho^{2}$, which, for the two values of $e^{2}$, gives two circles concentric with that one of the fixed circles which has its chord of contact parallel to the focal axis. Thus, for the two fixed circles, two pairs of circles constitute the locus.

Another case in which a reduction of degree occurs is when the two fixed conics are concentric, and the enveloping system 
has also the same centre. If we take the points $E, F$ at infinity in the tangential equation

$$
\theta^{2} E^{2}+F^{2}-2 \theta\left(A \lambda^{2}+B \mu^{2}+2 H \lambda \mu+C \nu^{2}\right)=0,
$$

where the origin is at the fixed centre, we may write

$$
E=l \lambda+m \mu, \quad F=l^{\prime} \lambda+m^{\prime} \mu,
$$

so that $E, F$ are the points at infinity on the sides of a parallelogram circumscribed about both the fixed conics. The foci are then determined by

$$
\begin{aligned}
& \theta^{2}(l+i m)^{2}+\left(l^{\prime}+i m^{\prime}\right)^{2}-2 \theta\left(A-B+2 i H+C u^{2}\right)=0, \\
& \theta^{2}(l-i m)^{2}+\left(l^{\prime}-i m^{\prime}\right)^{2}-2 \theta\left(A-B-2 i H+C v^{2}\right)=0,
\end{aligned}
$$

from which we obtain a result of the form

$$
a\left(x^{2}-y^{2}-\alpha^{2}\right)^{2}+b\left(2 x y-\beta^{2}\right)^{2}+c\left(2 x y-\beta^{2}\right)\left(x^{2}-y^{2}-\alpha^{2}\right)=d .
$$

If, further, the fixed conics are both inscribed in a rectangle, taking the axes parallel to the sides of the rectangle, we may write $E=\lambda, F=\mu$, so that the system becomes

$$
\theta^{2} \lambda^{2}+\mu^{2}-2 \theta\left(A \lambda^{2}+B \mu^{2}+C \nu^{2}+2 H \lambda \mu\right)=0,
$$

and the locus reduces to the equilateral hyperbola

$$
C x y+H=0 \text {. }
$$

Another method of arriving at the particular conditions subject to which the general sextic locus breaks up into two circular cubics may be indicated here.

Let the general system be written

$$
\begin{aligned}
& \theta^{2}\left(x_{1} \lambda+y_{1} \mu+\nu\right)^{2}+\left(x_{2} \lambda+y_{2} \mu+\nu\right)^{2} \\
& -\theta\left(A \lambda^{2}+B \mu^{2}+C \nu^{2}+2 F \mu \nu+2 G \nu \lambda+2 H \lambda \mu\right)=0
\end{aligned}
$$

in tangential coördinates, and suppose that one conic of the system is a circle. This evidently subjects the two given conics to a single condition. Then if the corresponding value of $\theta$ is $k$, and if we put $x_{1} \lambda+y_{1} \mu+\nu=E, x_{2} \lambda+y_{2} \mu+\nu=F$, we must have

$$
k E^{2}+\frac{1}{k} F^{2}-\left(A \lambda^{2}+\text { etc. }\right)=(l \lambda+m \mu+n \nu)^{2}+p\left(\lambda^{2}+\mu^{2}\right) ;
$$

for the equation on the right-hand side is the general tangential equation of a circle. Hence if $l \lambda+m \mu+n \nu=G$, which represents the centre of the circle, we get

$$
A \lambda^{2}+\text { etc. }=k E^{2}+\frac{1}{k} E^{2}-G^{2}-p\left(\lambda^{2}+\mu^{2}\right) .
$$

Thus the system written above becomes

$$
\theta^{2} E^{2}+F^{2}-\theta\left\{k E^{2}+\frac{1}{k} E^{2}-G^{2}-p\left(\lambda^{2}+\mu^{2}\right)\right\}=0 .
$$


Now two conics whose tangential equations differ by $\lambda^{2}+\mu^{2}$ (the tangential equation of $I, J$ ) are confocal. Hence the conic just written has the same foci as

$$
\theta^{2} E^{2}+F^{2}-\theta\left\{k E^{2}+\frac{1}{k} F^{2}-G^{2}\right\}=0 .
$$

But the envelope of the latter conic is

$$
\left(k^{2} E^{2}+F^{2}-k G^{2}\right)^{2}-4 k^{2} E^{2} F^{2}=0,
$$

which breaks up into the four factors

$$
k E \pm F \pm G \sqrt{k}=0 .
$$

Thus the envelope consists of four points forming a quadrilateral of which the points $E, F, G$ are the intersections of opposite sides and diagonals. Hence we have arrived at the following result: If two conics $U, V$ are such that a circle can be described to have double contact with both of them, then the foci of a system of conics having double contact with $U, V$ coincide with the foci of a conic passing through four fixed points. Of course it immediately follows from this that the locus in the one case is the same as that in the other. Now if $\rho_{1}, \rho_{2}, \rho_{3}, \rho_{4}$ are the distances of a point from the four fixed points and $l, m, n, p$ are the areas of the triangles formed by the latter, Sylvester showed that the locus is

$$
l_{\rho_{1}}+m \rho_{2}+n \rho_{3}+p \rho_{4}=0
$$

a curve of the sixth order, which agrees with what we have shown in this article. But, furthermore, he demonstrated that if the four points are concyclic, this locus breaks up into two circular cubics, of which the four points are foci. And this case would appear to correspond to that in which we have shown a similar breaking up to take place, viz. when two circles can be described to have double contact with the two fixed conics.

Another case of a similar kind may be added. Suppose the two fixed conics to have a focus in common, then if $\alpha, \beta, \gamma$ are the perpendiculars from the three foci on a line, we may write, in tangential coördinates,

$$
\Sigma=\frac{\alpha \beta}{b^{2}}-\Omega, \quad \Sigma^{\prime}=\frac{\alpha \gamma}{b^{\prime 2}}-\Omega,
$$

where $\Omega(=1)$ is the tangential equation of $I, J$. We have, then, for one of the systems of enveloping conics,

and $\quad \theta^{2} \alpha^{2}-2 \theta\left(\frac{\alpha \beta}{h^{2}}+\frac{\alpha \gamma}{b^{2}}-2 \Omega\right)+\left(\frac{\beta}{b^{2}}-\frac{\gamma}{b^{12}}\right)^{2}=0$.

$$
E F=\alpha\left(\frac{\beta}{b^{2}}-\frac{\gamma}{b^{\prime 2}}\right)
$$


But this conic is confocal with

$$
\theta^{2} \alpha^{2}-2 \theta \alpha\left(\frac{\beta}{b^{2}}+\frac{\gamma}{b^{12}}\right)+\left(\frac{\beta}{b^{2}}-\frac{\gamma}{b^{12}}\right)^{2}=0,
$$

which may be written

$$
\sqrt{\theta \alpha}+\frac{1}{b} \sqrt{\beta}+\frac{1}{b^{\prime}} \sqrt{\gamma}=0
$$

which represents a conic passing through $\alpha, \beta, \gamma$, and touching a given tangent at the first of these points. Now this is a particular case of the system of conics passing through four fixed points ; viz. it is obtained by supposing two of the points to coincide and determine a definite direction. Thus the locus is reduced to a particular case of Sylvester's result. Hence, also, it will follow that if the tangent

$$
\alpha=0, \frac{\beta}{b^{2}}-\frac{\gamma}{b^{12}}=0
$$

is the tangent to the circumscribing circle of $\alpha, \beta, \gamma$ at $\alpha$, then the locus breaks up into the two circular cubics of which $\alpha$ is the node and $\beta, \gamma$ the foci.

In the case of spherical conics there are somewhat similar results, but they are less simple and interesting, as necessarily follows from the fact that a spherical conic has, in addition to the two real foci, two pairs of imaginary ones; that is, not counting opposite points as different foci. This is in consequence of the points $I, J$ being replaced by the imaginary circle at infinity. Properties, however, which do not depend upon the absolute breaking up into factors remain the same. Thus if a system of sphero-conics be described to have double contact with a fixed sphero-conic $U$ and a fixed circle $V$, a pair of their foci (not necessarily real) will lie on a sphero-conic confocal with $U$ and passing through a pair of the intersections of the common tangent ares of $U$ and $V$.

Again suppose we consider a system of sphero-conics having double contact with two sphero-conies which both have double contact with another conic $\Sigma$. Writing the two conics

$$
\mathbf{\Sigma}=(P+Q)^{2}, \mathbf{\Sigma}=(P-Q)^{2},
$$

in tangential coördinates, so that $P, Q$ are intersections of common tangents, the system may be written

$$
\frac{P^{2}}{\cos ^{2} \theta}+\frac{Q^{2}}{\sin ^{2} \theta}=\Sigma \text {, }
$$

for the envelope of this with regard to $\theta$ breaks up into the factors $\Sigma=(P \pm Q)^{2}$. Now suppose, as we have already done for a particular case in plano, that $\mathrm{\Sigma}$ is a circle; then

$$
\Sigma=R^{2}-\Omega
$$


where $R$ is the centre, and $\Omega$ is the imaginary circle at infinity. Thus the system becomes

$$
\frac{P^{2}}{\cos ^{2} \theta}+\frac{Q^{2}}{\sin ^{2} \theta}=R^{2}-\Omega
$$

that is, a conic confocal with

$$
\frac{P^{2}}{\cos ^{2} \theta}+\frac{Q^{2}}{\sin ^{2} \theta}=R^{2}
$$

Thus the locus of the foci of the system coincides with the similar locus for a system of sphero-conics passing through four fixed points. The latter lies on a cone of the sixth degree, but when the four points are concyclic, does not break up into factors, as takes place in the plane case. This locus can easily be found as follows: let $\rho_{1}, \rho_{2}, \rho_{3}, \rho_{4}$ be the arcual distances of any arbitrary point of the sphere from four fixed points of the surface, then we can evidently determine $l, m$, $n, p$ so that the relation

$$
l \cos \rho_{1}+m \cos \rho_{2}+n \cos \rho_{3}+p \cos \rho_{4}=0
$$

is identically true. This is, in fact, the determinant relation

$$
\left|\begin{array}{rrrr}
\cos \rho_{1}, & \cos \rho_{2}, & \cos \rho_{3}, & \cos \rho_{4} \\
x_{1}, & x_{2}, & x_{3}, & x_{4} \\
y_{1}, & y_{2}, & y_{3}, & y_{4} \\
z_{1}, & z_{2}, & z_{3}, & z_{4}
\end{array}\right|=0 .
$$

Now let one focus satisfy this equation; then since the sum of the distances of a point of the curve from the foci is constant $=2 \alpha$, say, the other focus must satisfy

$$
\begin{gathered}
l \cos \left(2 \alpha-\rho_{1}\right)+m \cos \left(2 \alpha-\rho_{2}\right)+n \cos \left(2 \alpha-\rho_{3}\right) \\
+p \cos \left(2 \alpha-\rho_{4}\right)=0, \\
\text { or } \quad \cos 2 \alpha\left(l \cos \rho_{1}+m \cos \rho_{2}+n \cos \rho_{3}+p \cos \rho_{4}\right) \\
\quad+\sin 2 \alpha\left(l \sin \rho_{2}+m \sin \rho_{2}+n \sin \rho_{3}+p \sin \rho_{4}\right)=0,
\end{gathered}
$$

which in consequence of the identity reduces to

$$
l \sin \rho_{1}+m \sin \rho_{2}+n \sin \rho_{3}+p \sin \rho_{4}=0 .
$$

This, if cleared of radicals, would at first sight lead to a cone of the eighth degree, but it is easily seen to be divisible by $x^{2}+y^{2}+z^{2}$; for, putting

$$
\sin \rho_{1}=\sqrt{ }\left\{x^{2}+y^{2}+z^{2}-\left(x x_{1}+y y_{1}+z z_{1}\right)^{2}\right\}, \text { etc., }
$$

and then $x^{2}+y^{2}+z^{2}=0$, we get the identical relation.

If the four points are concyclic, we can easily find the 
resulting relation; for, putting $\rho_{1}=\rho_{2}=\rho_{3}=\rho_{4}$ for the centre of the circle in the identity, we get

$$
l+m+n+p=0 ;
$$

but combine this as we may with

$$
\Sigma l \cos \rho=0, \quad \Sigma l \sin \rho=0,
$$

it does not seem possible to demonstrate that the locus breaks up into factors.

With respect to the actual geometrical forms of the locus in the preceding cases, it would obviously be a work of great length and detail to enumerate and classify them in order. Besides considering the base conics with regard to their being elliptic or hyperbolic, and with regard to the reality or otherwise of their intersections, it would also be of primary importance to determine whether special forms of conics of the system existed, and whether they were real or imaginary. Thus if a parabola of the system is real, the locus has in general two real asymptotes, and if there is no real parabola, the locus has no real part at infinity. Similarly, if a circle belong to the system, its centre will give rise to a node on the locus, and, moreover, one at which the tangents are at right angles, as we see by considering the imaginary foci as well as the real. Further, in all cases the two pairs of common tangents of the base conics, into which two conics of the system break up for special values of the parameter, give rise to two nodes. The tangents at the latter are evidently the bisectors of the angle between the common tangents, and, if real, are the limiting states of the axes of hyperbolas.

It is to be observed that the locus, or part of the locus, may have no direct geometrical signification; that is, points may satisfy the equation of the locus without being foci of any real conic; for instance, the conic

$$
\frac{x^{2}}{a^{2}}+\frac{y^{2}}{b^{2}}+1=0
$$

which is entirely imaginary, envelopes the two real conics

$$
\begin{gathered}
\frac{a}{x}+\frac{b}{y}=1, \\
\left(\frac{x}{a}\right)^{\frac{1}{2}}+\left(\frac{y}{b}\right)^{\frac{1}{2}}=1,
\end{gathered}
$$

and has two real foci situated at $x=0, y^{2}=a^{2}-b^{2}$, where $a>b$. It seems thus that there may be a real locus for an entirely imaginary system of conics. 